\title{
On circular systematic sampling in the presence of linear trend
}

\begin{abstract}
The present paper deals with the computation of the circular systematic sample mean and its variance in the presence of linear trend among the population values. As a result, explicit expression for the variance of circular systematic sample mean is obtained for a pre-assigned fixed sample size and the population size. The efficiency of circular systematic sampling with that of simple random sampling without replacement is assessed algebraically and also for certain natural populations. It is observed that circular systematic sampling performs better than the simple random sampling without replacement.
\end{abstract}

Keywords: circular systematic sampling, linear systematic sampling, linear trend, optimal circular systematic sampling, simple random sampling, trend free sampling, yates type end corrections
Volume 7 Issue 4 - 2018

\section{Subramani J}

Department of Statistics, Pondicherry university, India

Correspondence: Subramani J, Department of Statistics, Pondicherry University, RV Nagar, Kalapet, Puducherry-605 014 , India,

Email drjsubramani@yahoo.co.in, drjsubramani@gmail.com

Received: April 13, 2018| Published: July 20, 2018

\section{Introduction}

Let $\boldsymbol{U}=\left(\boldsymbol{U}_{1}, \boldsymbol{U}_{2}, \ldots, \boldsymbol{U}_{\boldsymbol{N}}\right)$ be a finite population with $\boldsymbol{N}$ distinct and identifiable units and $\boldsymbol{Y}$ be a real variable with value $Y$. measured on $\boldsymbol{U}_{i}, \boldsymbol{i}=1,2, \ldots, \boldsymbol{N}$ giving a vector of measurements $\boldsymbol{Y}=\left(\boldsymbol{Y}_{1}, \boldsymbol{Y}_{2}, \ldots, \boldsymbol{Y}_{\boldsymbol{N}}^{\boldsymbol{i}}\right)$. If $\boldsymbol{Y}_{\boldsymbol{i}}=\boldsymbol{a}+\boldsymbol{i} \boldsymbol{b}, \boldsymbol{i}=1,2, \ldots, \boldsymbol{N}$ then the population is called a labelled population with a perfect linear trend among the population values. The problem is in general, to estimate the population mean $\bar{Y}=\frac{1}{N} \sum_{i=1}^{N} \boldsymbol{Y}_{i}$ on the basis of a sample of size ${ }^{\mathrm{n}}$ selected from the finite population $\mathrm{U}$. Any ordered sequence $\mathbf{S}=\left(\mathbf{u}_{1}, \mathbf{u}_{2}, \ldots, \mathbf{u}_{\mathbf{n}}\right)=\left(\mathbf{U}_{\mathbf{i} 1}, \mathbf{U}_{\mathbf{i} 2}, \ldots, \mathbf{U}_{\text {in }}\right), \quad 1 \leq i_{1} \leq N \quad$ and $\quad 1 \leq l \leq n$ is called a sample of size $n$. Several sampling schemes like, simple random sampling without replacement, systematic sampling are available in the literature for selecting a sample of fixed size $n$ from a finite population of size $N$. For the case of finite population with a linear trend among the population values and $N=k n$, the linear systematic sampling (LSS) is normally recommended for selecting a random sample of fixed size $n$. Further it is shown algebraically that the estimator from linear systematic sampling is better than the estimator provided by simple random sampling without replacement in the presence of linear trend. The performance of systematic sample mean can be improved further by introducing some modifications on the selection of the samples which includes the centered systematic sampling, ${ }^{1}$ balanced systematic sampling, ${ }^{2}$ modified systematic sampling ${ }^{3}$ and also by introducing changes in the estimator itself like, Yates type end corrections. ${ }^{4}$ In recent times several attempts are made to find an alternative to LSS. In this connection it is worth to note the following works which are alternative to LSS. Diagonal systematic sampling, ${ }^{5}$ Generalized diagonal systematic sampling, ${ }^{6,7}$ Determinant sampling, ${ }^{8}$ Modified linear systematic sampling, ${ }^{9-11}$ Generalized modified linear systematic sampling, ${ }^{12}$ Star type systematic sampling, ${ }^{13}$ Remainder linear systematic sampling, ${ }^{14}$ Generalized systematic sampling, ${ }^{15}$ Remainder linear systematic sampling, ${ }^{14}$ Modified balanced circular systematic sampling, ${ }^{16}$ Modified systematic sampling by Huang, ${ }^{17}$ Lahiri, ${ }^{18}$ Leu \& Tsui, ${ }^{19}$ Sampath \& Uthayakumaran, ${ }^{20}$ Singh \& Garg, ${ }^{21}$ Singh \& Singh, ${ }^{22}$ Uthayakumaran, ${ }^{23}$. For further discussions on linear systematic sampling the readers are referred to Cochran, ${ }^{24}$, Gautschi, ${ }^{25}$ Khan et al., ${ }^{26,27}$ Gupta \& Kabe, ${ }^{28}$ Murthy,,${ }^{29}$ Singh,${ }^{30}$ Sukhatme et al., ${ }^{31}$ Fountain

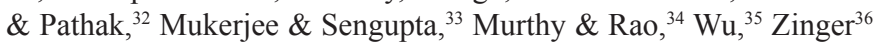
and the references cited there in.

If the population size $N$ is not a multiple of sample size $n(\mathrm{~N} \neq \mathrm{kn})$ then the linear systematic sampling is not applicable for selecting a sample of fixed size $n$. In such situations, circular systematic sampling (CSS) introduced by Lahiri ${ }^{18}$ cited in Murthy ${ }^{29}$ provides a constant sample size $n$ and the selected units are distinct if and only if $N$ and $k$ are relatively prime numbers. However the circular systematic samples are multiple copies of linear systematic samples when $N=k n$ and provides repetition of sampled units when N and $k$ are not relatively prime numbers. As pointed out by Subramani et al. ${ }^{13}$ the problems in circular systematic sampling are the following:

i. The choice for the sampling interval $\mathrm{k}$, which ensures the distinct units in the sample and minimum variance

ii. The explicit expressions for the variance of CSS sample mean which is useful to assess the efficiency of the CSS with other sampling schemes, particularly SRSWOR.

Several attempts have been made in the past to get a suitable value for the sampling interval for the given values of population size $N$ and sample size $n$. Murthy \& $\operatorname{Rao}^{34}$ has given the choice for $k$ as "It may be noted that the sample mean is unbiased for the population mean for all values of $k$, through the spread of the sample and hence efficiency is better if $k$ is taken as an integer nearest to $N / \mathrm{n}$. However, if repetition of the same unit in a sample is to be avoided, then it is desirable to take the sampling interval as $[N / \mathrm{n}]$ . It is shown that necessary and sufficient condition for all samples in CSS to have distinct units is that $N$ and $k$ are relatively co-prime". ${ }^{37}$ Bell house ${ }^{38-40}$ has suggested that the choice for the sampling interval $k=[(N / n)+(1 / 2)]$ when $N \neq(n-1) k$ and $k=(N / n)$ when $N=(n-1) k$.

Sengupta \& Chattopadhyay ${ }^{33}$ have proposed the following: "A necessary and sufficient condition for a circular systematic sampling of size n, drawn from a population of $N$ units with sampling interval $k$, to contain all distinct units is that $[N, \mathrm{k}] / k \geq n$ or equivalently, $N /(\mathrm{n}, k) \geq n$ where $[N, \mathrm{k}]$ and $(\mathrm{n}, k)$ denote respectively the least common multiple and the greatest common divisor of $N$ and $k$ ". 
However it seems there is no theoretical result or empirical study available to justify the choice of $k$ which ensures the efficient estimator or the estimator with minimum variance compared to other choices of $k$."

Recently Subramani et al., ${ }^{41}$ and Subramani $\&$ Singh $^{42}$ have made attempts to address the above problems and introduced the optimal circular systematic sampling (OCSS) together with the explicit expressions for its variance and the sampling interval $k$ in the presence of linear trend. In OCSS, the choice for the sampling interval $k$ is

$$
k n \bmod N= \pm 1
$$

where $k n \bmod N=-1$ represents $k n \bmod N=N-1$

For the hypothetical population with values $\mathrm{Y}_{\mathrm{i}}=\mathrm{a}+\mathrm{ib}, \mathrm{i}=1,2, \ldots, \mathrm{N}$,

The variance of OCSS sample mean is given as

$$
V\left(\bar{y}_{\text {ocss }}\right)=\frac{(N-1)(N+1)}{12 n^{2}} b^{2}
$$

The variance of SRSWOR sample mean is given as

$$
V\left(\bar{y}_{r}\right)=\frac{(N-n)(N+1)}{12 n} b^{2}
$$

Example 1.1: The procedure of obtaining the optimum value of $k$ is explained for the fixed values of sample size $n$ and the population size $N$.

$$
\begin{aligned}
& \text { If } N=12 \text { and } n=5 \text { then } k=7 . \text { That is } k n \bmod N=-1 \\
& \text { If } N=12 \text { and } n=5 \text { then } k=5 \text {. That is } k n \bmod N=+1
\end{aligned}
$$

The selected OCSS samples, their means, expected value and the variance are given for the sampling interval $k=7$ and $k=5$ in the following Table $1.1 \& 1.2$ :

Table I.I OCSS samples and their means for the sampling interval $k=7$

\begin{tabular}{lcccccc}
\hline Sample number & \multicolumn{3}{c}{ Sample values } & & OCSS mean \\
\hline 1 & 1 & 8 & 3 & 10 & 5 & 5.4 \\
2 & 2 & 9 & 4 & 11 & 6 & 6.4 \\
3 & 3 & 10 & 5 & 12 & 7 & 7.4 \\
4 & 4 & 11 & 6 & 1 & 8 & 6 \\
5 & 5 & 12 & 7 & 2 & 9 & 7 \\
6 & 6 & 1 & 8 & 3 & 10 & 5.6 \\
7 & 7 & 2 & 9 & 4 & 11 & 6.6 \\
8 & 8 & 3 & 10 & 5 & 12 & 7.6 \\
9 & 9 & 4 & 11 & 6 & 1 & 6.2 \\
10 & 10 & 5 & 12 & 7 & 2 & 7.2 \\
11 & 11 & 6 & 1 & 8 & 3 & 5.8 \\
12 & 12 & 7 & 2 & 9 & 4 & 6.8 \\
\hline
\end{tabular}

Table I.2 OCSS samples and their means for the sampling interval $k=5$

\begin{tabular}{lllllll}
\hline Sample Number & \multicolumn{3}{l}{ Sample Values } & & OCSS Mean \\
\hline 1 & 1 & 6 & 11 & 4 & 9 & 6.2 \\
2 & 2 & 7 & 12 & 5 & 10 & 7.2 \\
3 & 3 & 8 & 1 & 6 & 11 & 5.8 \\
4 & 4 & 9 & 2 & 7 & 12 & 6.8 \\
5 & 5 & 10 & 3 & 8 & 1 & 5.4 \\
6 & 6 & 11 & 4 & 9 & 2 & 6.4 \\
7 & 7 & 12 & 5 & 10 & 3 & 7.4 \\
8 & 8 & 1 & 6 & 11 & 4 & 6.0 \\
9 & 9 & 2 & 7 & 12 & 5 & 7.0 \\
10 & 10 & 3 & 8 & 1 & 6 & 5.6 \\
11 & 11 & 4 & 9 & 2 & 7 & 6.6 \\
12 & 12 & 5 & 10 & 3 & 8 & 7.6 \\
\hline
\end{tabular}

For both the cases of sampling interval $k=7$ and $k=5$, it is obtained that

$$
\begin{gathered}
V\left(\bar{y}_{\text {ocss }}\right)=\frac{1}{N} \sum_{i=1}^{N} \bar{y}_{i}^{2}-\bar{Y}^{2} \\
=\frac{512.72}{12}-6.5^{2}=42.72667-42.25=0.476667
\end{gathered}
$$

The value of the variance given above is coincided with the value obtained through the formula given in Table 1.2

Further it seems, no attempt is made to derive the explicit expression for the variance of circular systematic sample mean even after 65 years of its introduction for the case of labelled population with a perfect linear trend. As a consequence, the efficiency of circular systematic sampling is not assessed algebraically with that of simple random sampling without replacement.

The points noted above are motivating the present study, which deals with the following:

i. To derive the explicit expression for the variance of circular systematic sample mean for the population with a perfect linear trend among the population values

ii. To derive the explicit expressions for the Yates type end corrections for further improvements on the circular systematic sampling

iii. To assess the relative performance of circular systematic sampling with that of simple random sampling without replacement and the optimal circular systematic sampling algebraically and also for certain natural populations.

$i v$. To deduce the optimum values for the sampling fraction and the optimum variance for the circular systematic sampling.

\section{Circular systematic sampling}

As stated earlier, the LSS is not applicable when the population size $N$ is not a multiple of sample size $n(\mathrm{~N} \neq \mathrm{kn})$ for selecting a sample of fixed size $n$ whereas the CSS introduced by Lahiri ${ }^{18}$ cited in Murthy ${ }^{29}$ provides a constant sample size $n$. The steps involved in CSS for selecting a sample of size $n$ with sampling interval $k$ are given below: 
Step 1: Arrange the $N$ population units $\boldsymbol{U}=\left(\boldsymbol{U}_{1}, \boldsymbol{U}_{2}, \ldots, \boldsymbol{U}_{\boldsymbol{N}}\right)$ around a circle

Step 2: Select a random number $r$ such that $1 \leq r \leq N$

Step3: For selecting a circular systematic sample of size $n$ select every $k^{\text {th }}$ elements from the random start $r$ in the circle until $n$ elements are accumulated.

The selected units $\boldsymbol{U}_{\boldsymbol{r}}, \boldsymbol{U}_{\boldsymbol{r}+\boldsymbol{k}}, \boldsymbol{U}_{\boldsymbol{r}+\boldsymbol{k} \boldsymbol{k}}, \ldots, \boldsymbol{U}_{\boldsymbol{r}+(\boldsymbol{n}-1) \boldsymbol{k}}$ be the circular systematic sample of size $n$ for the random start $r$. If $\boldsymbol{r}+\boldsymbol{j} \boldsymbol{k}>\boldsymbol{N}$ then select the item corresponding to $\{\mathbf{r}+\mathbf{j k}-\mathbf{N}\}$

The variance of the circular systematic sample mean is obtained as given below:

$$
\boldsymbol{V}\left(\overline{\boldsymbol{y}}_{\text {css }}\right)=\frac{1}{\boldsymbol{N}} \sum_{i=1}^{N}\left(\overline{\boldsymbol{y}}_{\boldsymbol{i}}-\overline{\boldsymbol{Y}}\right)^{2}=\frac{1}{\boldsymbol{N}} \sum_{i=1}^{N} \overline{\boldsymbol{y}}_{\boldsymbol{i}}^{2}-\overline{\boldsymbol{Y}}^{2}
$$

Example 2.1: The procedure of obtaining the value of the sampling interval $k$ in the case of circular systematic sampling is explained for the fixed values of sample size $n$ and the population size $N$.

If $N=12$ and $n=5$ then $k=2$. That is

$$
\boldsymbol{k}=\boldsymbol{I n t}\left(\frac{N}{n}\right)=\operatorname{Int}\left(\frac{12}{5}\right)=2
$$

The selected CSS samples, their means, expected value and the variance are given for the sampling interval $k=2$ in the following Table 2.1.

Table 2.I CSS samples and their means for the sampling interval $k=2$

\begin{tabular}{lllllll}
\hline Sample number & \multicolumn{3}{c}{ Sample values } & & CSS mean \\
\hline 1 & 1 & 3 & 5 & 7 & 9 & 5 \\
2 & 2 & 4 & 6 & 8 & 10 & 6 \\
3 & 3 & 5 & 7 & 9 & 11 & 7 \\
4 & 4 & 6 & 8 & 10 & 12 & 8 \\
5 & 5 & 7 & 9 & 11 & 1 & 6.6 \\
6 & 6 & 8 & 10 & 12 & 2 & 7.6 \\
7 & 7 & 9 & 11 & 1 & 3 & 6.2 \\
8 & 8 & 10 & 12 & 2 & 4 & 7.2 \\
9 & 9 & 11 & 1 & 3 & 5 & 5.8 \\
10 & 10 & 12 & 2 & 4 & 6 & 6.8 \\
11 & 11 & 1 & 3 & 5 & 7 & 5.4 \\
12 & 12 & 2 & 4 & 6 & 8 & 6.4
\end{tabular}

For the cases of sampling interval $k=2$ and $n=5$, it is obtained that

$$
\begin{gathered}
V\left(\bar{y}_{c s s}\right)=\frac{1}{N} \sum_{i=1}^{N} \bar{y}_{i}^{2}-\bar{Y}^{2} \\
=\frac{515.6}{12}-6.5^{2}=42.96667-42.25=0.716667
\end{gathered}
$$

\section{Computation of circular systematic sample means}

Consider the labelled population with the $N$ population values $\boldsymbol{Y}_{\boldsymbol{i}}=\boldsymbol{a}+\boldsymbol{i b}, \boldsymbol{i}=1,2, \ldots, \boldsymbol{N}$.

The population mean is $\overline{\boldsymbol{Y}}=\boldsymbol{a}+\left[\frac{(\boldsymbol{N}+1)}{2}\right] \boldsymbol{b}(2.2)$

After a little algebra the circular systematic sample means are obtained as:

$$
\begin{gathered}
\bar{y}_{\mathbf{i .}}=\mathbf{a}+\left[\mathbf{i}+\frac{\mathbf{k}(\mathbf{n}-1)}{2}\right] \mathbf{b}, \quad \mathbf{i}=1,2,3 \ldots \mathbf{N}-\mathbf{k}(\mathbf{n}-1)=\mathbf{L}(\mathbf{s a y}) \\
\bar{y}_{\boldsymbol{i} .}=\boldsymbol{a}+\left[\boldsymbol{i}+\frac{\boldsymbol{k}(\boldsymbol{n}-1)}{2}-\frac{\boldsymbol{N}}{\boldsymbol{n}}\right] \boldsymbol{b}, \quad \boldsymbol{i}=\boldsymbol{L}+1, \boldsymbol{L}+2, \ldots, \boldsymbol{L}+\boldsymbol{k} \\
\overline{\boldsymbol{y}}_{\boldsymbol{i} .}=\boldsymbol{a}+\left[\boldsymbol{i}+\frac{\boldsymbol{k}(\boldsymbol{n}-1)}{2}-\frac{2 \boldsymbol{N}}{\boldsymbol{n}}\right] \boldsymbol{b}, \quad \boldsymbol{i}=\boldsymbol{L}+\boldsymbol{k}+1, \boldsymbol{L}+\boldsymbol{k}+2, \ldots, \boldsymbol{L}+2 \boldsymbol{k} \\
\bar{y}_{\boldsymbol{i} .}=\boldsymbol{a}+\left[\boldsymbol{i}+\frac{\boldsymbol{k}(\boldsymbol{n}-1)}{2}-\frac{3 \boldsymbol{N}}{\boldsymbol{n}}\right] \boldsymbol{b}, \quad \boldsymbol{i}=\boldsymbol{L}+2 \boldsymbol{k}+1, \boldsymbol{L}+2 \boldsymbol{k}+2, \ldots, \boldsymbol{L}+3 \boldsymbol{k} \\
\overline{\boldsymbol{y}}_{\boldsymbol{i} .}=\boldsymbol{a}+\left[\boldsymbol{i}+\frac{\boldsymbol{k}(\boldsymbol{n}-1)}{2}-\frac{(\boldsymbol{n}-1) \boldsymbol{N}}{\boldsymbol{n}}\right] \boldsymbol{b}, \quad \boldsymbol{i}=\boldsymbol{L}+(\boldsymbol{n}-2) \boldsymbol{k}+1, \boldsymbol{L}+(\boldsymbol{n}-2) \boldsymbol{k}+2, \ldots, \boldsymbol{L}+(\boldsymbol{n}-1) \boldsymbol{k}
\end{gathered}
$$

Remark 2.1: Since $\boldsymbol{L}=\boldsymbol{N}-\boldsymbol{k}(\boldsymbol{n}-1)$ then $\boldsymbol{L}+(\boldsymbol{n}-1) \boldsymbol{k}=\boldsymbol{N}$

From the above expressions the sum of the CSS sample means is obtained as

$$
\begin{aligned}
& \sum_{i=1}^{N} \bar{y}_{i}=\sum_{i=1}^{N}\left[a+\left[i+\frac{k(n-1)}{2}\right] b\right]-\frac{N b}{n} \sum_{j=1}^{n-1} \sum_{i=1}^{k} j \\
& \sum_{i=1}^{N} \bar{y}_{i}=N a+\left(\frac{N(N+1)}{2}+\frac{N k(n-1)}{2}\right) b-\frac{k N b n(n-1)}{2 n} \\
& \frac{1}{N} \sum_{i=1}^{N} \bar{y}_{i}=a+\left(\frac{N+1}{2}\right) b=\bar{Y}
\end{aligned}
$$

That is, the CSS sample mean is an unbiased estimator for its population mean.

\section{Computation of variance of circular systematic sample mean}

For the labelled population and the corresponding CSS sample means defined in Section 2.1, the derivation of the variance of circular systematic sample mean is given below.

Consider

$$
\begin{aligned}
& \boldsymbol{V}\left(\overline{\boldsymbol{y}}_{c s s}\right)=\boldsymbol{E}\left(\overline{\boldsymbol{y}}_{c s s}-\overline{\boldsymbol{Y}}\right)^{2} \\
& =\frac{1}{\boldsymbol{N}} \sum_{i=1}^{N} \overline{\boldsymbol{y}}_{i}^{2}-\overline{\boldsymbol{Y}}^{2}
\end{aligned}
$$


By substituting the CSS sample means and the population means in the above expression, the variance of CSS sample mean for the labelled population is obtained as

$$
V\left(\bar{y}_{c s s}\right)=\frac{b^{2}}{N}\left\{\begin{array}{l}
\sum_{i=1}^{N}\left[i+\frac{k(n-1)}{2}\right]^{2}+\frac{N^{2}}{n^{2}} \sum_{j=1}^{n-1} \sum_{i=1}^{k} j^{2}- \\
\frac{2 N}{n} \sum_{j=1}^{n-1} \sum_{i=L+(j-1) k+1}^{L+j k} j\left(i+\frac{k(n-1)}{2}\right)-\frac{N(N+1)^{2}}{4}
\end{array}\right\}
$$

After a little algebra, the variance of CSS sample mean is obtained as

$V\left(\bar{y}_{c s s}\right)=\frac{b^{2}}{N}\left\{\begin{array}{l}\frac{N(N+1)(2 N+1)}{6}+\frac{N k^{2}(n-1)^{2}}{4}+\frac{2 k(n-1) N(N+1)}{4}+\frac{k N^{2}(n-1) n(2 n-1)}{6 n^{2}}- \\ \frac{k N(n-1)[6 N+k(n-2)+3]}{6}-\frac{N(N+1)^{2}}{4}\end{array}\right\}$

By simplifying the above expression one may get

$$
\boldsymbol{V}\left(\bar{y}_{\text {css }}\right)=\left\{\frac{(\boldsymbol{N}-1)(\boldsymbol{N}+1)}{12}-\frac{\boldsymbol{k}\left(\boldsymbol{n}^{2}-1\right)(2 \boldsymbol{N}-\boldsymbol{k n})}{12 \boldsymbol{n}}\right\} \boldsymbol{b}^{2}
$$

\section{Computation of optimum values for the sampling fraction $k$ and the variance of circular systematic sample mean}

We know that the sampling fraction $\boldsymbol{k}$ is obtained as $\boldsymbol{k}=\boldsymbol{I n t}\left(\frac{\boldsymbol{N}}{\boldsymbol{n}}\right)$ or the positive integer closest to $\frac{\boldsymbol{N}}{\boldsymbol{n}}$. Without loss of generality, let us assume that $\boldsymbol{N}=\boldsymbol{k n} \pm \boldsymbol{d}$. That is $\boldsymbol{d}$ is the difference between $\boldsymbol{N}$ and $\boldsymbol{k n}$ and $\boldsymbol{k}=(\boldsymbol{N} \mp \boldsymbol{d}) / \boldsymbol{n}$. By replacing the values of $\boldsymbol{k}$ in the variance expression, one may get

$$
\begin{aligned}
& \boldsymbol{V}\left(\overline{\boldsymbol{y}}_{\text {css }}\right)=\left\{\frac{(N-1)(N+1)}{12}-\frac{\boldsymbol{k}\left(\boldsymbol{n}^{2}-1\right)(2 N-\boldsymbol{k n})}{12 \boldsymbol{n}}\right\} \boldsymbol{b}^{2} \\
& =\left\{\frac{(N-1)(N+1)}{12}-\frac{(N \mp \boldsymbol{d})\left(n^{2}-1\right)(2 N-(N \mp \boldsymbol{d}))}{12 \boldsymbol{n}^{2}}\right\} \boldsymbol{b}^{2} \\
& =\left\{\frac{(N-1)(N+1)}{12}-\frac{(N \mp d)\left(n^{2}-1\right)(N \pm d)}{12 n^{2}}\right\} b^{2} \\
& =\left\{\frac{(N-1)(N+1)}{12}-\frac{\left(n^{2}-1\right)\left(N^{2}-d^{2}\right)}{12 n^{2}}\right\} b^{2}
\end{aligned}
$$

By simplifying the above expression one may get

$$
V\left(\bar{y}_{c s s}\right)=\left\{\frac{(N-1)(N+1)}{12 n^{2}}+\frac{\left(n^{2}-1\right)\left(d^{2}-1\right)}{12 n^{2}}\right\} b^{2}
$$

The above expression attains minimum at $\boldsymbol{d}^{2}= \pm 1$, which implies

$$
\boldsymbol{N}=\boldsymbol{k n} \pm 1 \text { or } \boldsymbol{k n}=\boldsymbol{N} \pm 1 .
$$

That is, the optimum variance of CSS sample mean is exactly the same as given in (1.2).

$$
\boldsymbol{V}\left(\bar{y}_{\text {ocss }}\right)=\left\{\frac{(N-1)(N+1)}{12 n^{2}}\right\} b^{2}
$$

Hence we conclude that the optimum value of the sampling fraction $\boldsymbol{k}$ is obtained as $\boldsymbol{k n} \boldsymbol{m o d} \boldsymbol{N}= \pm 1$ stated by Subramani et al., ${ }^{41}$ and Subramani \& $\operatorname{Singh}^{42}$ as given in (Table 1.1)

\section{Comparison of the efficiency of CSS and SRSWOR sample means}

By comparing the variance expressions for a SRSWOR sample mean (1.3) and a CSS sample mean (2.7) one can easily show that

$$
\begin{gathered}
V\left(\bar{y}_{r}\right)-V\left(\bar{y}_{c s s}\right)=\frac{(N-n)(N+1) b^{2}}{12 n}-\left\{\frac{(N-1)(N+1)}{12 n^{2}}-\frac{\left(n^{2}-1\right)\left(d^{2}-1\right)}{12 n^{2}}\right\} b^{2} \\
=\left\{\frac{(N-n)(N+1)}{12 n}-\frac{(N-1)(N+1)}{12 n^{2}}+\frac{\left(n^{2}-1\right)\left(d^{2}-1\right)}{12 n^{2}}\right\} b^{2} \\
=\left\{\frac{(N+1)(n-1)(N-n+1)}{12 n^{2}}+\frac{\left(n^{2}-1\right)\left(d^{2}-1\right)}{12 n^{2}}\right\} b^{2} \geq 0
\end{gathered}
$$

That is, the circular systematic sampling is more efficient than the simple random sampling without replacement. i.e. $\boldsymbol{V}\left(\overline{\boldsymbol{y}}_{\text {css }}\right) \leq \boldsymbol{V}\left(\overline{\boldsymbol{y}}_{\boldsymbol{r}}\right)$

\section{Some modifications on circular systematic sample mean}

It has been shown in Section 3 that the circular systematic sampling performs better than the simple random sampling without replacement. However it is not a trend free sampling ${ }^{33}$ which can be achieved by introducing Yates type end corrections ${ }^{4}$ as given below:

The modification involves the usual circular systematic sampling but the modified sample mean is defined as

$$
\bar{y}_{c s s}^{*}=\bar{y}_{c s s}+a\left(y_{1}-y_{n}\right)
$$

That is, the units selected first and last are given the weights $\mathbf{n}^{-1}+\mathbf{a}$ and $\mathbf{n}^{-1}-\mathbf{a}$ respectively whereas the remaining units get the weight $\mathbf{n}^{-1}$. By equating $\overline{\boldsymbol{y}}_{\text {css }}^{*}=\overline{\boldsymbol{Y}}$ for the population with a perfect linear trend, we get the values for a from (4.1) as:

Here one may have the following two situations: (i). The random start $\boldsymbol{i}$ is less than or equal to $\boldsymbol{L}$ and (ii). The random start $\boldsymbol{i}$ is greater than $\boldsymbol{N}-\boldsymbol{k}(\boldsymbol{n}-1)=\boldsymbol{L}$.

Case (i). When the random start $\boldsymbol{i}$ is less than or equal to $\boldsymbol{N}-\boldsymbol{k}(\boldsymbol{n}-1)=\boldsymbol{L}$

By setting (4.1) is equal to $\overline{\boldsymbol{Y}}$ we get

$$
\left[\boldsymbol{i}+\frac{\boldsymbol{k}(\boldsymbol{n}-1)}{2}\right]+a\left(\boldsymbol{y}_{1}-\boldsymbol{y}_{\boldsymbol{n}}\right)=\frac{(\boldsymbol{N}+1)}{2}, \boldsymbol{i}=1,2, \ldots, \boldsymbol{N}-\boldsymbol{k}(\boldsymbol{n}-1)=\boldsymbol{L}
$$


By putting $\left(\mathbf{y}_{1}-\mathbf{y}_{\mathbf{n}}\right)=\mathbf{i}-(\mathbf{i}+(\mathbf{n}-1) \mathbf{k})=-\mathbf{k}(\mathbf{n}-1)$, (4.2)

we get $\boldsymbol{a}=\frac{2 \boldsymbol{i}+\boldsymbol{k}(\boldsymbol{n}-1)-(\boldsymbol{N}+1)}{2 \boldsymbol{k}(\boldsymbol{n}-1)}, \boldsymbol{i}=1,2,3, \ldots, \boldsymbol{N}-k(n-1)=L$

Case (ii). When the random start $\boldsymbol{i}$ is greater than $\boldsymbol{N}-\boldsymbol{k}(\boldsymbol{n}-1)=\boldsymbol{L}$

Let the random start $\boldsymbol{i}$ lies between $\boldsymbol{L}+(\boldsymbol{j}-1) \boldsymbol{k}+1$ and $\boldsymbol{L}+\boldsymbol{j} \boldsymbol{k}$

By setting (4.1) is equal to $\overline{\boldsymbol{Y}}$ we get

$\left[i+\frac{k(n-1)}{2}-\frac{j N}{n}\right]+a\left(y_{1}-y_{n}\right)=\frac{(N+1)}{2}, i=L+(j-1) k+1$

to $L+j k$ get

By putting $\left(\mathbf{y}_{1}-\mathbf{y}_{\mathbf{n}}\right)=\mathbf{i}-(\mathbf{i}+(\mathbf{n}-1) \mathbf{k}-\mathbf{j} \mathbf{N})=\mathbf{j} \mathbf{N}-\mathbf{k}(\mathbf{n}-1)$ we

$$
\mathbf{a}=\frac{\boldsymbol{n}[(\boldsymbol{N}+1)-2 \boldsymbol{i}-\boldsymbol{k}(\boldsymbol{n}-1)]+2 \boldsymbol{j} \boldsymbol{N}}{2 \boldsymbol{n}[\boldsymbol{j} \boldsymbol{N}-\boldsymbol{k}(\boldsymbol{n}-1)]}, \boldsymbol{i}=\boldsymbol{L}+(\boldsymbol{j}-1) \boldsymbol{k}+1 \text { to }
$$

$$
L+j k
$$

Remark 4.1: In the presence of a perfect linear trend the modified circular systematic sample mean $\overline{\mathbf{y}}_{\text {css }}^{*}$ becomes the population mean $\overline{\boldsymbol{Y}}$ and hence the $\mathrm{V}\left(\bar{y}_{\mathrm{css}}^{*}\right)=0$. In this case the circular systematic sampling becomes a completely trend free sampling (See Mukerjee and Sengupta, 1990).

\section{Numerical comparisons of circular systematic sampling for certain natural populations}

It has been shown in Section 3 that the circular systematic sampling performs well compared to simple random sampling without replacement whenever there exists a perfect linear trend among the population values. However this is an unrealistic assumption in real life situations. Consequently an attempt has been made to study the efficiency of the circular systematic sampling for a population considered by Subramani ${ }^{6}$ and Murthy ${ }^{29}$ The first data were collected for assessing the process capability of a manufacturing process from an auto ancillary manufacturing unit located in Tamilnadu. The data pertain to the measurements taken continuously during the Turning operation performed on the component Torsion bar in Frontier CNC Lathe Machine. The data were collected for estimating the mean value of the outer diameter of the Torsion bar, one of the key components in integrated power steering system. The measurements were taken continuously for the first 50 components produced in a shift. The 50 measurements based on the order of the production are given in Table 3.1. However the first 37 measurements after arranging the data in ascending order are taken to get a linear trend among the population values as given in Table 3.2. The Second data are about the number of workers for 80 factories in a region. However the first 37 measurements of the data are taken to get a linear trend among the population values as given in Table 3.3.

The variances of simple random sample mean, circular systematic sample mean and optimal circular systematic sample mean together with the percentage relative efficiencies are obtained and are presented in Table 3.4. The PREs of the proposed estimator $(\boldsymbol{p})$ with respect to an existing estimator $(\boldsymbol{e})$ is computed as

$$
\operatorname{PRE}(p)=\frac{V(e)}{V(p)} \times 100
$$

It is seen from the table values that the optimal circular systematic sampling performs better than the circular systematic sampling and the circular systematic sampling performs better than simple random sampling in all the cases. In general, it is observed that for the population with a linear trend the following inequality is true.

That is, $\boldsymbol{V}\left(\overline{\boldsymbol{y}}_{\text {ocss }}\right) \leq \boldsymbol{V}\left(\overline{\boldsymbol{y}}_{\text {css }}\right) \leq \boldsymbol{V}\left(\overline{\boldsymbol{y}}_{\boldsymbol{r}}\right)$

Table 3.I Data of outer Diameter of Torsion Bar (Spec.9065 \pm 25 )

\begin{tabular}{llllllllll}
\hline 9050 & 9052 & 9050 & 9052 & 9052 & 9056 & 9056 & 9054 & 9056 & 9058 \\
9054 & 9054 & 9060 & 9058 & 9060 & 9058 & 9056 & 9058 & 9058 & 9060 \\
9062 & 9064 & 9062 & 9064 & 9066 & 9070 & 9068 & 9072 & 9072 & 9070 \\
9072 & 9070 & 9070 & 9072 & 9074 & 9076 & 9078 & 9076 & 9076 & 9078 \\
9078 & 9078 & 9082 & 9080 & 9082 & 9080 & 9082 & 9086 & 9086 & 9084
\end{tabular}

Table 3.2 The data arranged in ascending order

\begin{tabular}{llllllllll}
\hline 9050 & 9050 & 9052 & 9052 & 9052 & 9054 & 9054 & 9054 & 9056 & 9056 \\
9056 & 9056 & 9058 & 9058 & 9058 & 9058 & 9058 & 9060 & 9060 & 9060 \\
9062 & 9062 & 9064 & 9064 & 9066 & 9068 & 9070 & 9070 & 9070 & 9070 \\
9072 & 9072 & 9072 & 9072 & 9074 & 9076 & 9078 & & &
\end{tabular}

Table 3.3 Number of workers in first 37 factories (Murthy, 1967, p.228)

$\begin{array}{llllllllll}51 & 51 & 52 & 52 & 53 & 54 & 57 & 60 & 65 & 67 \\ 68 & 70 & 71 & 73 & 74 & 76 & 78 & 80 & 81 & 85 \\ 87 & 88 & 92 & 93 & 97 & 100 & 107 & 110 & 113 & 116 \\ 119 & 121 & 125 & 127 & 127 & 131 & 134 & & & \end{array}$


Table 3.4 Comparison of optimal circular systematic sampling, circular systematic sampling and simple random sampling without replacement

\begin{tabular}{|c|c|c|c|c|c|c|c|c|c|c|c|}
\hline \multirow{2}{*}{$\mathbf{N}$} & \multirow{2}{*}{$\mathbf{n}$} & \multicolumn{5}{|c|}{ Population I } & \multicolumn{5}{|c|}{ Population 2} \\
\hline & & & & & PRE- & PRE- & & & & PRE- & PRE- \\
\hline 37 & 2 & 30.59 & 15.95 & 15.95 & 191.81 & 100 & 320.89 & 176.87 & 176.87 & 181.43 & 100 \\
\hline 37 & 3 & 19.81 & 6.03 & 6.03 & 328.49 & 100 & 207.81 & 68.09 & 68.09 & 305.2 & 100 \\
\hline 37 & 4 & 14.42 & 4.96 & 4.96 & 290.76 & 100 & 151.28 & 35.99 & 35.99 & 420.34 & 100 \\
\hline 37 & 5 & 11.19 & 3.15 & 2.93 & 381.51 & 107.37 & 117.35 & 25.19 & 22.69 & 517.19 & 111.02 \\
\hline 37 & 6 & 9.03 & 1.81 & 1.81 & 498.07 & 100 & 94.74 & 15.68 & 15.68 & 604.21 & 100 \\
\hline 37 & 7 & 7.49 & 1.7 & 1.66 & 451.2 & 102.11 & 78.59 & 13.74 & $12.0 \mid$ & 654.37 & 114.4 \\
\hline 37 & 8 & 6.34 & 1.51 & 1.51 & 420.44 & 100 & 66.47 & 13.17 & 10.12 & 656.82 & 130.14 \\
\hline 37 & 9 & 5.44 & 0.81 & 0.81 & 673.73 & 100 & 57.05 & 7.57 & 7.57 & 753.63 & 100 \\
\hline 37 & 10 & 4.72 & 1.01 & 0.76 & 620.11 & 132.72 & 49.51 & 10.02 & 5.44 & 910.11 & 184.19 \\
\hline 37 & II & 4.13 & 4.13 & 0.64 & $64 I .46$ & 641.46 & 43.34 & 13.13 & 5.67 & 764.37 & 231.57 \\
\hline 37 & 12 & 3.64 & 3.64 & 3.64 & 100 & 100 & 38.2 & 4.73 & 4.73 & 807.61 & 100 \\
\hline 37 & 13 & 3.23 & 3.23 & 0.37 & 865.15 & 865.15 & 33.85 & 5.55 & 3.85 & 879.22 & 144.16 \\
\hline 37 & 14 & 2.87 & 2.87 & 0.39 & 743.78 & 743.78 & 30.12 & 11.05 & 2.86 & 1053.15 & 386.36 \\
\hline 37 & 15 & 2.56 & 0.23 & 0.23 & 1095.3 & 100 & 26.89 & 27.12 & 2.68 & 1003.36 & 1011.94 \\
\hline 37 & 16 & 2.29 & 1.37 & 0.3 & 754.61 & 449.67 & 24.07 & 14.57 & 2.5 & 962.8 & 582.8 \\
\hline 37 & 17 & 2.06 & 0.62 & 0.21 & 993.24 & 300 & 21.57 & 6.14 & 1.72 & 1254.07 & 356.98 \\
\hline 37 & 18 & 1.85 & 0.24 & 0.24 & 781.78 & 100 & 19.36 & 1.88 & 1.88 & 1029.79 & 100 \\
\hline 37 & 19 & 1.66 & 0.21 & 0.21 & 781.13 & 100 & 17.37 & 1.69 & 1.69 & 1027.81 & 100 \\
\hline 37 & 20 & 1.49 & 0.45 & 0.15 & 990.67 & 298.67 & 15.59 & 4.43 & 1.24 & 1257.26 & 357.26 \\
\hline
\end{tabular}

\section{Acknowledgements}

The Author wishes to record his heartiest thanks for the Editor and the Reviewer for their constructive comments, which have improved the presentation of the paper.

\section{Conflict of interest}

The author declares that no conflicts.

\section{References}

1. Madow WG. On the theory of systematic sampling III. Comparison of centered and random start systematic sampling. Ann Math Statist. 1953;24(1):101-106.

2. Sethi VK. On optimum pairing of units. Sankhya B. 1965;27(3-4):315320.

3. Singh D, Jindal KK, Garg JN On modified systematic sampling. Biometrika. 1968;55(3):541-546.

4. Yates F Systematic Sampling. Phil Trans Royal Soc A. 1948;241(834):345377.

5. Subramani J. Diagonal Systematic Sampling Scheme for Finite Populations. Journal of the Indian Society of Agricultural Statistics. 2000;53(2):187-195.

6. Subramani J. Further results on diagonal systematic sampling for finite populations. Journal of the Indian Society of Agricultural Statistics. 2009;63(3):277-282.
7. Subramani J. Generalization of Diagonal Systematic Sampling Scheme for Finite Populations. Model Assisted Statistics and Applications. 2010;5(2):17-128.

8. Subramani J, Tracy DS. Determinant Sampling Scheme for Finite Populations. Internl J Math \& Statist Sci. 1999;8(1):27-41

9. Subramani J. A modification on linear systematic sampling for odd sample size. Bonfring International Journal of Data Mining. 2012;2(2):32-36.

10. Subramani J. A Modification on Systematic Sampling. Model Assisted Statistics and Applications. 2013;8(3):215-227.

11. Subramani J. A Further Modification on Systematic Sampling for Finite Populations. Journal of Statistical Theory and Practice. 2013;7(3):471479.

12. Subramani J, Gupta SN. Generalized modified linear systematic sampling scheme for finite populations. Hacettepe Journal of Mathematics and Statistics (HJMS). 2014;43(3):529-542.

13. Subramani J. Star Type Systematic Sampling Schemes for Finite Populations. Communications in Statistics-Theory and Methods. 2014;43(1):175-190.

14. Chang HJ, Huang KC. Remainder linear systematic sampling. Sankhya B. 2000;62(2):249-256.

15. Khan Z, Shabbir J, Gupta S. A New Sampling Design for Systematic Sampling. Communications in Statistics-Theory and Methods. 2013;42(18): 3359-3370.

16. Ching Ho Leu, Fei-Fei Kao. Modified balanced circular systematic sampling. Statistics \& Probability Letters. 2006;76:373-383. 
17. Huang KC. Mixed random systematic sampling designs. Metrika. 2004;59(1):1-11.

18. Lahiri DB. A method of sample selection providing unbiased ratio estimates. Ball Internat Statist Inst. 1951;33:33-140.

19. Leu CH, Tsui KW. New partially systematic sampling. Statist Sinica. 1996;6:617-630.

20. Sampath S, Uthayakumaran N. Markov systematic sampling. Biometrical J. 1998;40(7):883-895.

21. Singh P, Garg JN. On balanced random sampling. Sankhya C. 1979;41:6068.

22. Singh D, Singh P. New systematic sampling. JSPI. 1977;1:163-177.

23. Uthayakumaran N. Additional circular systematic sampling methods. Biometrical J. 1998;40(4):467-474.

24. Cochran WG. Sampling Techniques, $3^{\text {rd }}$ ed. New York: John Wiley and Sons; 1977.

25. Gautschi W. Some remarks on systematic sampling. AMS. 1957;28:385394.

26. Khan Z, Gupta S, Shabbir J. Diagonal Circular Systematic Sampling, to appear in Journal of Statistical Theory and Practice. 2013.

27. Khan Z, Gupta S, Shabbir J. Generalized Systematic Sampling. Journal of Communications in Statistics-Simulation and Computation. 2013;44(9): 2240-2250.

28. Gupta AK, Kabe DG. Theory of Sample Surveys. World Scientific Publishing Company. 2011:236.

29. Murthy MN. Sampling Theory and Methods. India: Statistical Publishing House; 1967.

30. Sarjinder Singh. Advanced Sampling Theory with Applications. Springer; 2003.
31. Sukhatme PV, Sukhatme BV, Sukhatme S, et al. Sampling Theory of Surveys with Applications. USA: Iowa State Univ Press; 1984. p. 1-519.

32. Fountain RL, Pathak P. Systematic and Non-random Sampling in the Presence of Linear Trends. Communications in statistics-Theory and Methods. 1989;18(7):2511-2526.

33. Mukerjee R, Sengupta S. Optimal Estimation of a Finite Population Means in the Presence of Linear Trend. Biometrika. 1990; 77(3):625-630.

34. Murthy MN, Rao TJ. Systematic sampling with illustrative examples. In: Krishnaiah PR, Rao CR, editors. Handbook of Statistics. 1988;6:147-185.

35. WU CFJ. Estimation in systematic sampling with supplementary observations. Sankhya B. 1984;46:306-315.

36. Zinger A. Variance estimation in partially systematic sampling. JASA. 1980;75(369):206-211.

37. Sudakar K. A note on circular systematic sampling. Sankhya C. 1978;40:72-73.

38. Bell House DR. On the choice of sampling interval in circular systematic sampling. Shankya B. 1984;247-248.

39. Bell House DR. Systematic sampling. In: Krishnaiah PR, Rao CR, editors. Handbook of Statistics. 1988;6:125-145.

40. Bell House DR, Rao JNK. Systematic sampling in the presence of linear trends. Biometrika. 1975;62(3):694-697.

41. Subramani J, Gupta SN, Prabavathy G.): Circular systematic sampling in the presence of linear trend. American Journal of Mathematical and Management Sciences (AJMMS). 2014;33(1):1-19.

42. Subramani J, Sarjinder Singh. Estimation of Population Mean in the Presence of Linear Trend. Communications in Statistics-Theory and Methods. 2014;43(15):3095-3116. 